\title{
What Role, if any, Should Economic Evaluation Play in Market Access Decisions of Pharmaceutical Treatments for Cancer Patients with Short Life Expectancy?
}

I believe that economic evaluation plays an important and essential role in the market access decisions of pharmaceutical treatments for cancer patients with a short life expectancy. Increasing health care expenditures, a rapid introduction of new medical technologies has led to a growing interest in information from economic evaluations for decision making about resource allocation in health care. Economic evaluations can provide valuable information in relation to drug use, other healthcare intervention/programmes, and investments in research and new technologies. However, there are currently the most widely used pricing and reimbursement decisions for new drugs and health technology assessments of cancer patients. ${ }^{1}$

An example of economic evaluation in market access decisions of pharmaceutical treatments for cancer patients with short life expectancy in the UK is valuebased pricing (VBP), which is a potential solution to barriers to accessing new expensive drugs for healthcare providers, and to improve market access/reimbursement for the industry. Examples of VBP include cost-effectiveness analysis (CEA) and therapeutic added value (TAV). CEA tries to determine the value of spending on medicines or policies that are returned to sufferers, such as longer life and better quality of life. ${ }^{2}$

The CEA of drugs is assessed for reimbursement price using QALY and incremental costs relative to existing treatments. QALYs are calculated by multiplying how much a given treatment will lengthen a patient's life by improving the quality. CEA for drugs is regarded by economists as being, in theory, compatible with efficient resource allocation. TAV assessments typically include a relationship with other instituted medications in the same class used in the standard of care. Higher costs settled for improved health or different values recognized by payers. ${ }^{2}$

Health economics and outcomes researchers generally measure value using the CEA tool with the QALY as the health gain measure. ${ }^{3}$ Most health technology assessment (HTA) systems base their decision-making on QALY. Health care systems should recognize a wide range of cost savings in the present and the future if they result directly from interventions of interest, as recommended by the second panel on cost-effectiveness in health and medicine. $^{3}$

The benefit is measured from the patient's perspective addressed by healthcare technology in question. Ideally, QALY can be used to measure the health gains of any technology. Costs and QALYs frequently form the basis of CEA value assessments. The QALY continues to be seen as a measure of the medical benefits to be used in population decisionmaking. ${ }^{3}$

Figure 1 shows the incorporation of additional elements of value into CEA (the preferred method of showing value), where twelve potential elements of value are considered. Four of them - productivity, net costs, adherence-improving factors, and QALYare traditionally included in value assessments. Eight others currently have novelty in economic assessments, such as disease severity, the value of hope, fear of contagion, reducing uncertainty, insurance value, equity, real option value, and scientific spillover. Many of these theoretical elements of value are well known and are ready for incorporation in value assessments, with the difference of two exceptions: scientific spillover and equity, which so far have not the required attention, possibly because they require more consensus and theoretical improvement. ${ }^{4}$ These elements are important for the economic evaluation of end-of-life care patients.

Up to $150,000 /$ QALY can be spent on end-of-life pharmaceutical products. It differs from other pharmaceutical products for the general population, which have a lower value, such as £500o/QALY or much less. For example, the management of chronic pain remains a challenge for physicians. Opioids are the main source of treatment for chronic severe pain. 


\section{Elements of Value}

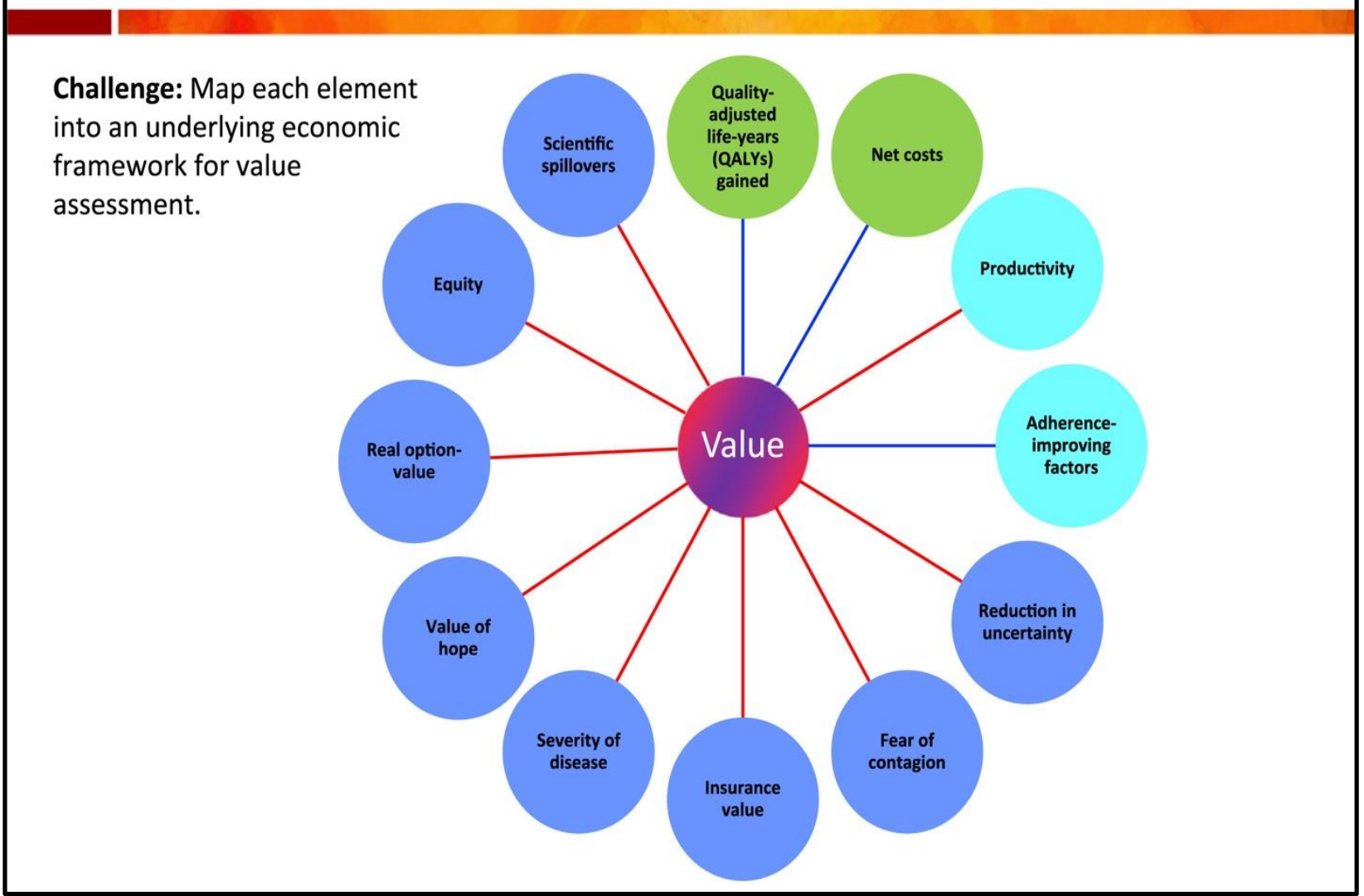

Figure 1. Twelve elements of value incorporated into CEA. ${ }^{4}$

A recent meta-analysis showed that tapentadol PR (TAP PR) and oxycodone/naloxone (OXN) both drugs have a better tolerability profile than a traditional opioid, such as oxycodone CR. OXN gained in one year 0.29 QALYs compared to its counterpart OXY CR. ${ }^{5}$

End-of-life care drugs have a higher QALY compared to other pharmaceutical products for the general population. Many innovative end-of-life drugs are biologics, such as monoclonal antibodies that have a high cost of production. Previous rigorous research and clinical trials have made them even more expensive. Therefore, their QALY thresholds are much higher than those of other pharmaceutical products for the general population.

I agree with the idea of the existence of an 'end-oflife' premium for such treatments with monthly affordable price contributions to health insurance institutions that agree to cover any palliative care, medical treatment, or diagnostic interventions for end-of-life care of patients. This would facilitate the use of a large amount of money to pay for drugs and may release the NHS hardship that would otherwise have had to contribute to the treatment using the highest QALY thresholds to purchase medicines for the pharmaceutical industry.

The insurance would pay for drugs that give an extra few months of life expectancy to terminally ill patients, and it helps to prepare patients' families psychologically for the short span of life of their loved ones. On the other hand, for a shorter life span period and overall survival insurance could also contribute to other alternative medicine care to decrease the burden of end-of-life care for patients and their families.

In January 2019, a voluntary scheme for branded medicines and access (VPAS) was introduced to replace the UK Pharmaceutical Price Regulation 
Scheme (PPRS). This scheme aims to strike a balance between supporting innovation in the pharmaceutical industry, helping to get the most cost-effective medicines to patients as quickly as possible, and ensuring complete predictability of spending for the entire branded medicines bill for the NHS. The VPAS means that the branded medicines bill will not grow by more than $2 \%$ in any of the next five years. ${ }^{6}$

The government predicts that this will end in profits of $\pm 930 \mathrm{~m}$. Nevertheless, further steps to expedite decision-making on new medications suggest that they could reach sufferers up to six months more quickly than today. The aforementioned low rate of growth, coupled with new cost controls launched by NHS England on new innovative medicines, signifies that the pharmaceutical division is 'not dancing for satisfaction' over the deal. However, it provides stability and predictability for the United Kingdom sector, where the doubts of Brexit remain to endanger the overall UK marketability. ${ }^{7}$

The cost-benefit evaluation of health interventions should be analyzed in all modalities of treatment, whether palliative or pharmaceutical. It is essential to assess differences, if any, that may exist in the economic evaluation of such treatments. Likewise, QALY as a part of CEA is assessed for all treatments and health interventions as an example that economic evaluation of non-pharmaceutical interventions/initiatives in palliative care or end-oflife care should not differ from pharmaceutical treatments.

I consider that buying expensive drugs to extend endof-life patients should also be linked to the ability of the drugs to improve the quality of life of those with end-of-life diseases. New policies should be developed in the application of more palliative care, pain management, and use of alternative and traditional medicine modalities to ameliorate the suffering of many of these end-of-life conditions versus increasing the QALY threshold for purchasing expensive drugs, with little information on extending or improving quality of life. It is understood that all deserve the best treatment at any stage of their disease. This finding is significant for patients with rare cancers. Therefore, the use of orphan drugs is essential. However, monetary resources should be allocated to improve the existing programs on vaccinations in childhood. In addition, nationwide vaccination programs should be implemented to prevent the emergence of diseases. The NHS should achieve comprehensive cancer screening programs for the most common cancers that affect humans as breast, lung, prostate, and colorectal cancers, or purchasing or developing new treatments for new diseases such as COVID-19. Public perceptions can be aligned to NICE policies by recommendations on lower prices of medicines for the end of life and improve people's quality of life. A lower QALY threshold for end-of-life drugs is made cheaper and more widely available, with easy access to the sick.

\section{REFERENCES}

1. Lundkvist J. The role of economics in health care. 2005 (Online Thesis). Available at: https://openarchive.ki.se/xmlui/bitstream/handle/106 $16 / 39993 /$ thesis.pdf? sequence $=1$. [Last accessed on $25^{\text {th }}$ May,2021]

2. Towse A, Barnsley P. Approaches to identifying, measuring, and aggregating elements of value. Int J Technol Assess Health Care. 2013;29(4):360-4. https://doi.org/10.1017/So266462313000524.

3. Garrison LP Jr, Neumann PJ, Willke RJ, Basu A, Danzon PM, Doshi JA, et al. A Health Economics Approach to US Value Assessment FrameworksSummary and Recommendations of the ISPOR Special Task Force Report. Value Health. 2018;21(2):161-5.

https://doi.org/10.1016/j.jval.2017.12.009.

4. Lakdawalla DN, Doshi JA, Garrison LP Jr, Phelps CE, Basu A, Danzon PM. Defining Elements of Value in Health Care-A Health Economics Approach: An ISPOR Special Task Force Report. Value Health. 2018;21(2):131-9.

https://doi.org/10.1016/j.jval.2017.12.007.

5. Coluzzi F, Ruggeri M. Valutazione clinica ed economica di nuovi analgesici per la gestione del dolore cronico [Clinical and economical evaluation of new analgesics for the management of chronic pain]. Recenti Prog Med. 2014;105(11):415-9. (In Italian). https://doi.org/10.1701/1680.18402.

6. The Association of the British Pharmaceutical Industry. What is the new Voluntary Scheme on branded medicines? (Online Article). Available at: https://www.abpi.org.uk/new-medicines/medicinepricing-in-the-uk/what-is-the-new-voluntary-schemeon-branded-medicines/ [Last accessed on 25th May,2021]

7. PMLive. The UK's new five-year pricing agreement (Online Article). Available from): http://www.pmlive.com/pharma_intelligence/The_ 
UKs_new_five-year_pricing_agreement_1274601.

[Last accessed on $25^{\text {th }}$ May,2021]

Cite this article as:

Justiz-Vaillant A. What Role, if any, Should Economic Evaluation Play in Market Access

Decisions of Pharmaceutical Treatments for Cancer Patients with Short Life Expectancy?.

Int Healthc Res J. 2021;5(3):SC1-SC4. https://doi.org/10.26440/IHRJ/0503.06421

\section{AUTHOR AFFILIATIONS:}

Department of Para-Clinical Sciences. University of the West Indies. St. Augustine Campus. Trinidad and Tobago

(https://orcid.org/oooo-0oo2-3486-1963)

e-mail id for correspondence: angel.vaillant[at]sta[dot]uwi[dot]edu 